\title{
Shock refraction from classical gas to relativistic plasma environments
}

\author{
Rony Keppens, Peter Delmont and Zakaria Meliani \\ Centre for Plasma Astrophysics, K.U.Leuven, \\ Celestijnenlaan 200B, 3001 Heverlee, Belgium \\ email: Rony. Keppens@wis .kuleuven . be
}

\begin{abstract}
The interaction of (strong) shock waves with localized density changes is of particular relevance to laboratory as well as astrophysical research. Shock tubes have been intensively studied in the lab for decades and much has been learned about shocks impinging on sudden density contrasts. In astrophysics, modern observations vividly demonstrate how (even relativistic) winds or jets show complex refraction patterns as they encounter denser interstellar material.

In this contribution, we highlight recent insights into shock refraction patterns, starting from classical up to relativistic hydro and extended to magnetohydrodynamic scenarios. Combining analytical predictions for shock refraction patterns exploiting Riemann solver methodologies, we confront numerical, analytical and (historic) laboratory insights. Using parallel, gridadaptive simulations, we demonstrate the fate of Richtmyer-Meshkov instabilities when going from gaseous to magnetized plasma scenarios. The simulations invoke idealized configurations closely resembling lab analogues, while extending them to relativistic flow regimes.
\end{abstract}

Keywords. MHD, shock waves, instabilities

\section{Introduction}

Classical shock tube experiments have been conducted extensively, and in their most basic setup, they realize the laboratory counterpart of the theorist's Riemann problem, where two (static) gases are first separated by a membrane which is then suddenly removed. By controlling the pressure and density conditions on either side of the membrane, such in essence 1D problems have a known analytic solution. In case a high pressure gas is left to expand into a low pressure part, three signals arise, consisting typically of a rarefaction wave, contact discontinuity (CD), and shock wave as the high pressure gas sweeps up shocked matter in its sudden invasion of the low pressure medium. In fact, the Riemann problem solution is known under the more general situation where two states of arbitrary velocity, pressure and density are in contact, and its solution forms a major component of many contemporary shock-capturing numerical discretizations.

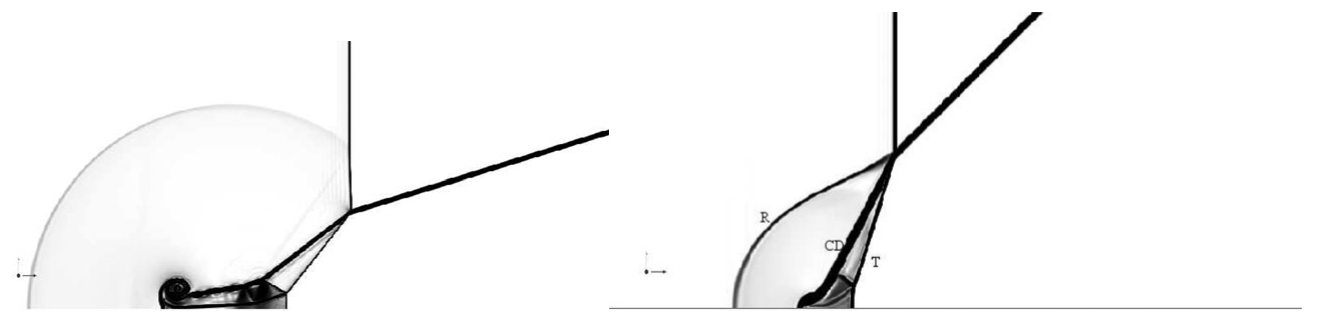

Figure 1. Irregular (left) versus regular (right) refraction pattern. A Schlieren plot of the density (i.e. an exponentially stretched quantification of the density gradient magnitude) is shown. The lower boundary is reflective. 
In a more general refraction setup, one considers a (rectangular) tube with a membrane at arbitrary inclination angle $\alpha$ to the horizontal, seperating two static gases which differ in density. This density contrast is quantified as $\eta$, with $\eta<1$ indicating a density decrease, or so-called slow-fast contact discontinuity, while $\eta>1$ is referred to as a fast-slow case. This nomenclature relates to the corresponding change in sound speed. A refraction problem is then created by using a piston to generate a shock of given strength (i.e. Mach number $M$ ) at the left hand end of the tube, which traverses the tube and interacts, or refracts, with this initial contact interface. From extensive experimental campaigns, a whole zoology of shock refraction patterns is known (Abd-El-Fattah \& Henderson, 1978). A recurring aspect, irrespective of whether the impinging shock is (very) weak to strong, is the transition from an irregular to a regular refraction pattern, when the angle $\alpha$ is varied from shallow to large values (up to $90^{\circ}$ inclination angles, at which point the problem is a $1 \mathrm{D}$ shock-CD interaction problem). Fig. 1 illustrates both an irregular (left) and a regular (right) refraction, in a 2D hydrodynamic simulation where $M=2, \eta=3$ is a fast-slow transition. The figure shows a Schlieren plot of the density, and the only parameter varied is the inclination angle $\alpha$. In a regular refraction case, the reflected (R), shocked contact (CD) and transmitted signal (T) meet in a single triple point at the point where the shock (vertical signal) passes over the initial contact (rightmost signal).

\section{From Newtonian to relativistic hydro refractions}

The specific case of regular refractions still collects several refraction patterns, since the reflected signal can be discontinuous (shock) or be of the continuous expansion fan variety. In the $1 \mathrm{D}$ case when $\alpha=90^{\circ}$, this is still understood from the known solution to the standard Riemann problem, as usually described in $x-t$ phase space. Indeed, as the shock then meets the (then aligned) initial contact discontinuity, the postshock state comes into contact with the state behind this CD, in turn realizing a standard Riemann problem. The (a) slow-fast versus (b) fast-slow transitions then coincide with the distinction where (a) the reflected signal is an expansion fan, while the transmitted signal moves ahead of the shock; versus (b) a case where the reflected signal is also of shock-type, and the transmitted signal lags the initial shock speed. Even when the setup is truly $2 \mathrm{D}$ (with $0^{\circ}<\alpha<90^{\circ}$ ), the regular refraction is amenable to exact analytic solution, as one realizes that in the frame comoving with the triple point, one has a timeindependent Riemann problem in the $x-y$ plane to solve. This $2 \mathrm{D}$ planar hydrodynamics refraction, and the complete Riemann-solver based solution strategy to predict critical angles for irregular to regular refractions, as well as full predictions for the 2D density, pressure and velocity variations, is described in detail in Delmont et al. (2009). Using the predictions from this solution strategy, one can quantify exactly the numerical accuracy for simulated refraction patterns in this regular refraction phase. This also allows to quantify the amount of vorticity deposited on the shocked contact discontinuity, which is the ultimate regulating factor in the further non-linear evolution where the RichtmyerMeshkov instability ensues. Indeed, as a jump in tangential velocity is created across the shocked contact, slight perturbations of this interface will be Kelvin-Helmholtz unstable, causing a complex rippling of the shocked CD. This instability can also occur when the speeds involved are tending towards astrophysically relevant, relativistic speeds. In Fig. 2, a snapshot of a $2 \mathrm{D}$ relativistic hydro case is visualized, where a Mach $M=100$ shock impinged on a fast-slow $\eta=1000$ transition, while the postshock velocity corresponded to half the speed of light. The angle $\alpha=45^{\circ}$ is no longer obviously detectable at this time, but one can see how the darker colored regions demark the region between the 
(fully Richtmyer-Meshkov deformed) CD and the transmitted shock front. The intricate wave and shock patterns behind these regions correspond to the at this point multiply reflected waves, complicated by both top and bottom solid wall interactions. They each induce novel (triple) point interactions, with slip lines becoming unstable to local Kelvin-Helmholtz activity. The amount of detail visible here is due to the grid-adaptive capabilities of the MPI-AMRVAC software (van der Holst \& Keppens, 2007) used in this study, where for this particular relativistic hydro run, 7 refinement levels realize an effective resolution of $1536 \times 7680$. Despite this high resolution, the full simulation completes within day(s) on a local desktop PC.

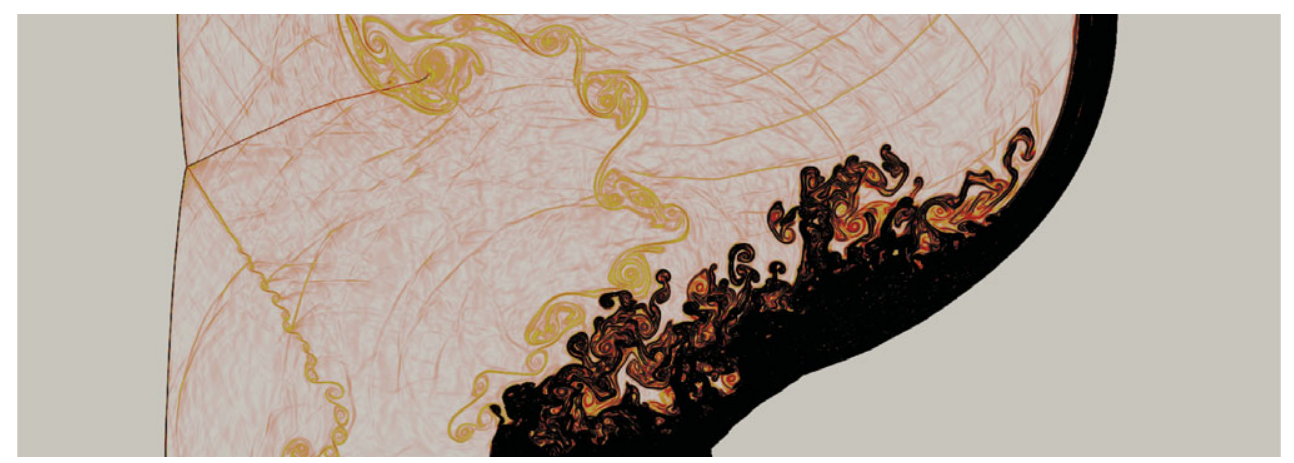

Figure 2. The late evolutionary stage of a relativistic hydro refraction problem. After a regular refraction phase, the shocked contact has become Richtmyer-Meshkov unstable. The reflected signal has by now had multiple interactions with the top and bottom reflective boundaries, as highlighted by this Schlieren density plot.

\section{Planar MHD regular refraction}

When the same setup is realized in a plasma environment, the macroscopic dynamics is governed by the magnetohydrodynamic (MHD) conservation laws. If a magnetic field purely perpendicular to the simulated plane (hence parallel to the shock front) is considered, it is possible to show that similar to the pure hydrodynamic case, three signals arise at refraction, and the ultimate instability to Richtmyer-Meshkov development is virtually identical for varying plasma beta conditions, as long as the Atwood number of the impinging shock (i.e. its density contrast) is kept fixed (Delmont et al., 2009).

The MHD case under a general magnetic field configuration is more complex due to the presence of multiple reflected, as well as transmitted, wave signals. If we restrict the situation to planar MHD, where the magnetic field is initially purely normal to the shock front and uniform throughout, the shock-CD interaction will give rise to 5 signals, and a quintuple point appears in a regular refraction situation. Once more, this case is amenable to analytic treatment, where the exact variation of all state variables (density, pressure, velocity and magnetic field) about the quintuple point is predicted. One can obtain self-similar solutions where in essence all quantities vary only with polar angle $\phi$ about the quintuple point, and across shocks standard MHD Rankine-Hugoniot relations apply. The latter relations immediately indicate an important difference between the (planar) MHD versus the hydro case: no vorticity jump is allowed across the shocked contact, and the perturbed contact discontinuity is no longer Richtmyer-Meshkov unstable. Still, depending on governing parameters, the $2 \mathrm{D}$ refraction problem can show intricate transitions in its precise shock refraction dynamics. This relates to the fact that MHD shock types come in several varieties, depending on wether one crosses slow, Alfvén or fast characteristic speeds. An example transition is shown in Fig. 3, where an $M=2$, $\eta=3$ fast-slow case (as in Fig. 1) is compared for plasma beta $\beta=0.5$ (left) to $\beta=4$ 


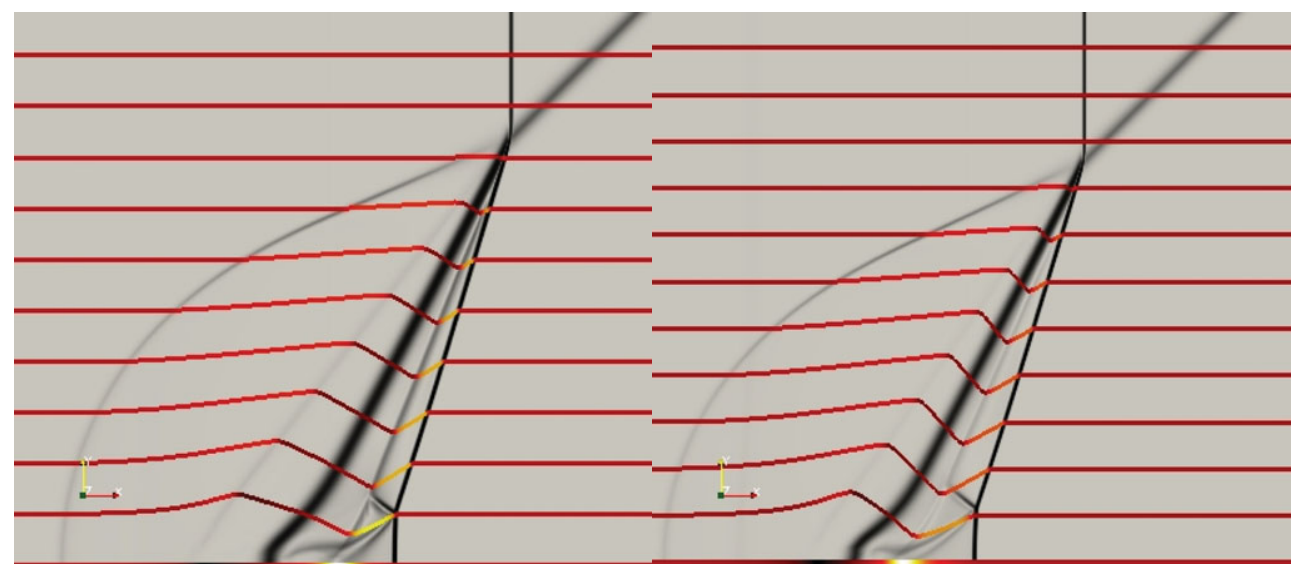

Figure 3. Planar MHD shock refraction leads to 5 signals in the regular refraction case. Left: for strong magnetic field $(\beta=0.5)$. Right: for weaker field $(\beta=4)$. The transmitted and reflected slow signals then change from slow shocks (left) to intermediate shocks (right).

(right) scenarios. This change relates to an overall decrease in the strength of the field, and the fieldlines together with the Schlieren density plots are shown. By quantifying the change in angle of the fieldlines with respect to the local shock normal, one concludes that the higher field case contains a fast shock, slow shock, CD, slow shock, fast shock refraction pattern. The lower field case at right rather has a fast shock, intermediate shock (crossing the Alfvén speed), CD, intermediate and fast shock pattern. It is to be stressed that these, and other subtle changes in the refraction pattern behavior can be quantified and predicted exactly using the Riemann solver based methodology, and that the numerical experiments as shown in Fig. 3, confirm these predictions precisely (Delmont \& Keppens, 2010).

\section{Outlook}

Shock refractions in both gas and plasma dynamical setups provide a stringent test for shock-instability dominated dynamics. Using grid-adaptive simulations, pattern transitions and the further nonlinear developments can be studied in all details. Analytic knowledge of the solution verifies the role of magnetic fields, and one can explore the transition to speeds where relativistic effects become essential. The information gathered from laboratory experiments helps to validate code efforts, and it is easily recognized how refractions (i.e. shock front interactions with pre-existing density changes) form an essential part in many astrophysically motivated problems where jets, winds or more violent explosion fronts encounter denser or more rarified molecular cloud regions.

\section{References}

Abd-El-Fattah, A. M. \& Henderson, L. F. 1978, JFM 86, 15

Delmont, P., Keppens, R. \& van der Holst, B. 2009, JFM, 627, 33

Delmont, P. \& Keppens, R. 2010, J. Phys. Conf Ser., 216, 012007

van der Holst, B. \& Keppens, R. 2007, JCP, 226, 925 